\title{
Linguistics in the Study and Teaching
} of Literature

\section{Nigel Fabb*}

University of Strathclyde

\section{Abstract}

Literary texts include linguistic form, as well as specialized literary forms (some of which also involve language). Linguistics can offer to literary studies an understanding of these kinds of form, and the ways by which a text is used to communicate meaning. In order to cope with the great variety of creative uses of language in literature, linguistics must acknowledge that some texts are assigned structure by non-linguistic means, but the boundaries between linguistic and non-linguistic explanations for literary language are not clearly drawn. The article concludes with discussion of what kinds and level of linguistics might usefully be taught in a literature classroom, and offers practical suggestions for the application of linguistics to literature teaching.

\section{The Oddity of Literary Language}

It would seem obvious that as linguists we have a special role in the teaching of literature, because we are experts in the medium - language - from which literary texts are made. However, linguists do not have a monopoly on discussion or theorization of language in literary studies. Poets and writers, particularly in their manifestos or statements about practice, make various statements about the language of poetry which ignore or deny what linguists know about language. One characteristic claim is that a particular writing practice involves 'a new syntax' or that a poet 'creates a new language'. The poet Ece Ayhan talks about '. . . pushing Turkish syntax overboard, eliding or "misplacing" syntactically connective tissues, so that words lose their linear context and explode from an unsettled center' (Rothenberg and Joris 1998: 484). Helmut Heissenbüttel describes his own practice as 'connections are made not through systematic and logico-syntactical interweavings, but through connotations, through ambiguities - outgrowths of a decayed syntax' (Rothenberg and Joris 1998: 164). This is a metaphorical use of terms, such as 'syntax' or 'language', and it is not uncharacteristic of literary studies, where there is a wish for another type of language than the everyday language, a language in which new types of meaning can be communicated. How, then, can 
we as linguists deal with these claims, and the texts from which they emerge, and more generally speak to our colleagues who are scholars of literature?

In this article, I assume a model of cognition, which most linguists will recognize, in which cognitive processes are computations (represented by rules and constraints) over symbols (or 'representations'). Some of these cognitive processes are specialized for language (e.g. syntax, phonology, and lexicon) and constitute the language faculty. A second type of cognitive process can be thought of as 'general'; these are processes that are not specific to particular domains, and might include general logical rules by which one thought is derived from another (like modus ponens), or general principles of analogy, or simple combinatory rules. One of the major types of literary linguistics now active (particularly in metaphor studies), called 'cognitive poetics', is committed to the idea that the mind (including language) is organized entirely by general processes (Turner 1996). In contrast to 'cognitive poetics', I would like to hold open the possibility that not only do we have language faculty processes that are specific to language, and also general processes, but also there might be other specific cognitive processes for particular purposes relevant to literature and language (such as the processing of poetic meter, or narrative form). These are the type of process identified, for example, by Tooby and Cosmides (1992), or Sperber (1996, 2000), or Mithen (1996), or Boyer (2001), among others (the underlying notion of 'massive modularity' is attacked by Fodor 2000, and defended by Carruthers 2006).

As an example of the third type of specialized process, consider metrical verse. A metrical text is a text that is divided into lines, such that each of the lines is measured (crudely, has a specific number of syllables). In addition, in almost all meters, the syllables are partitioned into two classes, and the placement of the syllable relative to other syllables in the line can depend on the class of the syllable; classical Greek meters, for example, distinguish between light and heavy syllables and control for the presence of one or the other. In Fabb and Halle (2008), we explain these two connected properties of metrical texts by formulating a universal set of parametrized rules that are specialized to process poetic meter. Each meter is built from this universal set and generates from the line a certain type of representation (a grid consisting of asterisks and parentheses); the grid is generated step-by-step, and can be further subject to rules altering its structure. Conditions then connect the partitioning of syllables with the grid (e.g. requiring heavy syllables to be in particular grid positions). The rules (and representations) involved are not part of language in general, but they are specific to one use of language, which is meter. Perhaps other aspects of literary cognition are devolved to specialized rule systems (modules) in this way.

In the 'culture and cognition' literature (Sperber, Mithen, Boyer, etc.), various specialized modules have been proposed - for processing information 
about animals, or for stablizing religious concepts, for example. Similarly, there might be a module that assigns genres to texts, or one that divides narratives into the orientation-complication-resolution-coda structure, or one that divides narratives into episodes, or one that divides a text (not necessarily metrical) into lines, verse paragraphs and other sections. In Fabb (2002), I argued that only general processes of inference are involved in these assignments of literary form to texts, based on general logical rules or notions of resemblance; that is, literary forms are concepts corresponding to categories and best understood in terms of prototypes or exemplars (Murphy 2002). However, I now think that the formal complexity of many of these types of literary form, and the systematicity and widespread similarities in the forms involved, might better be understood by thinking of them as terms in domain-specific computations: as involving distinct sets of symbols, processed by distinct rules and conditions. I will refer to them as 'modularized' and specifically as being central modules, to distinguish them from Fodorian input modules (Fodor 1983), including the parts of the language faculty.

These central modules are not specifically 'linguistic': they are not just part of the language faculty and might be inactive in everyday verbal behaviour. However, many of the modules for the assignment of literary form must have some access to structural descriptions assigned by the linguistic systems. For example, metrical rules must be able to identify syllables, and certain phonological characteristics of syllables. It has been argued that rhyme can 'see' underlying representations of sounds in some cases (Fabb 1997: 127). The determination of narrative form is often dependent on linguistic elements. Furthermore, the modules might share sub-components: Fabb and Halle (2008) argue that the same type of iterative rule is found in the language faculty (assignment of stress in words), in musical cognition, and in poetic metrical cognition.

Linguists have studied the symbols and rules/constraints operating in all three areas: in the language faculty, in central cognition (e.g. the study of pragmatics), and in specialized central modules (e.g. linguistic studies of meter or narrative), and linguistics offers much insight into their nature and operations. Given that literature draws on all three kinds of cognition, this suggests that linguistics has a special role in the study and teaching of literature.

The linguistic modules assign structural descriptions to data, each instance of which is an instance of (public) language. Where the data have been taken from actual speech, or writing, perhaps from a corpus, it may be necessary to correct the data: for example, in assigning syntactic structural descriptions, we might first remove hesitations, false starts, speech errors (although these might be kept in the data if we were for example looking at conversational structure). But literature presents a problem. Consider, for example, the poem 'Chinese Creep' by Kenward Elmslie, which includes the word 'tlooth' (in Ashbery 1974). This is presented by the text 
as a word of English (i.e. we are not being asked to read it as an imported foreign word), but it is not a word of English and, furthermore, cannot be assigned a structural description by the syllable structure rules of English. If this were non-literary data, we might treat it as a slip of the tongue or some other performance matter, and idealize it by correcting it to 'tooth', which can be assigned a structural description. But in the literary text, the word is as it is and not the corrected version; hence, we are caught on the one hand between recognizing the deliberate choice of a word which cannot be assigned a structural description and on the other hand our need to assign a structural description to the word so that we can deliver it to further computational processes. Similar examples can be found in all literature. For example, it is common in poetry for phrases to be reordered, without clear functional motivation (i.e. no obvious topicalization of the items involved; just movement). Thus, we find 'But past is all his fame' (Goldsmith), or 'And happier they their happiness who knew' (Shelley), sentences for which structural descriptions cannot be assigned by the syntactic rules, because they require transformations which are unmotivated or impossible.

Another kind of problem is presented by texts where words are combined in lists, and not clearly combined by any syntactic means at all; do we correct these texts by first formulating sentences based on them, and then subjecting these sentences to the syntactic rules which assign them structural descriptions? Austin (1984) explores various ways of handling the oddity of literary language. One possibility which some linguists endorse is that there is a different syntax for literary texts (e.g. there might be a syntax in which movement is free rather than functionally driven); a radical variant of this was proposed by Thorne (1965) who proposed that each poem might have its own grammar. Another possibility is that the language faculty assigns structural description to whatever fragments or parts of the text it can (e.g. to isolated phrases), but that the phrases are concatenated by general cognitive processes, not by syntactic rules. The larger texts so produced may resemble sentences but they are not as wholes assigned structural descriptions by the syntax, and the syntax provides no explanation of why they take the form they do. For example, a sequence by Pope, 'on her white breast a sparkling cross she wore', cannot be directly assigned a structural description as a whole by the syntax. This is for two reasons. First, there is no obvious motivation for movement of the phrases - they are not obviously topicalized, but rather appear to have just been reordered 'for stylistic reasons' (which could include fitting the required metrical pattern, clearly not a syntactically formulable requirement). Under minimalist approaches to syntax, phrases do not move unless they are forced to by some feature; hence, the syntax should not be able to move these phrases. Second, movement of the object to between subject and verb is an illegitimate movement generally in English; so both structurally and informationally, the sentence is not derivable by the syntactic rules. 
It is always worth remembering that the text of a literary work can be produced by non-linguistic means, even if it is made of linguistic material. Borges's (1998) 'Library of Babel' contains on its shelves books, whose totality constitutes every possible combination of the letters of the alphabet - hence, contains all actual books and all possible books. The copy of Pride and Prejudice in this library was not composed by linguistic means, even though it contains what look like actual words and sentences; in this library, it is an accidental combination of a sequence of letters. Less alarmingly, many literary texts in practice are composed by simple (nonlinguistic) processes of concatenation and juxtaposition of component parts; a poem consisting just of nouns has no syntactic structure. These extreme examples are not divided by any clear boundary from 'ordinary' literary texts, and in Fabb (2004), I raise the possibility that the language of any literary text might be a copy or representation of language, rather than 'the real thing'. If this is true, then we can never take for granted that any part of a text should be assigned a structural description.

\section{Meaning}

In the literature classroom, the dominant concern is to establish 'what a text is about', either at the large scale of understanding the meanings of a story, or in a smaller way establishing the ambiguous meanings of a poem, or how a metaphor is used. Many teachers of literature are happy for students to pursue their own interpretations of the text. In part, this comes from the recognition which literary studies share with linguistic theory that the realities of how communication works are such that the speaker/writer's meaning can never be finally established (short of telepathy). But in part the literature teacher's relatively relaxed view of interpretation comes also from a romantic view that literature produces meanings which can in some sense be perceived or apprehended but cannot be represented by being put into words. A large part of the literary ambition (cited in the previous section) to 'create a new language' has as its goal the expression of these new and otherwise ungraspable or unstateable kinds of meaning, which are outside our ordinary systems and modes of explanation (i.e. beyond the capacity of linguistic theory to explain). If we are to teach semantics to literature students, we need to give some respect to this view that there are meanings that are beyond us. Ideally, we want to be able to teach students a semantics that helps them understand some of the mechanisms by which meaning is communicated, without closing off some of the more inspiring notions of meaning that come from literary theory and the ideas of poets. Two potential areas of exploration include Sperber and Wilson's notion of 'poetic effects' that are produced by the generation of a large number of weak implicatures, as in a metaphor or evocation (Sperber and Wilson 1995), and Sperber's proposals about the rationality of apparently irrational beliefs (Sperber 1985); for further discussion, see Fabb (1995). 


\section{Appropriate Use of Linguistic Theory in the Literature Classroom}

In this section, I discuss the terminology that we use for describing the language of a literary text. It is best illustrated with an example. In seventeenth-century poetry, certain types of imagery are called 'conceits'; these are metaphors that are often conventional (i.e. similar metaphors can be found in earlier and classical literature; Ruthven 1969) and deployed over an extended part of a text, often as part of a structured argument. The term 'conceit' is a word of English, known to practitioners and useful in understanding how those practitioners imagined their own literary practice. We might call it a 'Rhetorical Term', because it is part of a known and formalized poetics (a rhetoric). If we take a relevancetheoretic approach to conceits, we might instead understand them as a particular exploitation of the principle of relevance; we might say that 'conceit' is not a well-formed notion at this level, and not useful in understanding how metaphors are processed in the mind, and we might introduce the term 'implicature' in discussing this, and give it the technical meaning specific to relevance theory (where an implicature is an intended implication or contextual assumption). The term 'implicature' would be a 'theoretical term': it exists only as a component of the theory itself. Literary scholars tend to take what might be called an ethnographic approach and work with rhetorical terms, while linguists tend to take a universalizing or mentalistic approach and work with theoretical terms. In the teaching of literature from a linguistic perspective, a middle way is useful, as I now explain.

When I teach about metaphor (in my class on semantics for literature students), I teach Grice's four maxims, and the co-operative principle, and I expect the students to be able to identify which of the maxims is being flouted; in a conceit, the maxim of quality is almost certainly flouted (as more generally in metaphors). I teach this, despite having no theoretical commitment to the notion of maxims; in fact, I take the relevancetheoretic position that the maxims can be dispensed with entirely. So for me, the term 'maxim of quality' is a 'useful term': it is neither part of a general rhetoric nor of any theory that I have a commitment to, but I find it useful. Why, then, teach something that I (strictly) do not believe? I do so because I think students need a fairly rich terminology for description, and as a way of understanding how texts work; a good theory reduces its terminology as much as possible (by Occam's razor), but this is not necessarily good for the classroom. The maxims are relatively easy to understand; although the difference between the four maxims is problematic, this is also a stimulus to discussion. In contrast, I find that the theoretically most convincing account provided by relevance theory gives too little for the student to grasp hold of and work with, and it is too difficult without extensive study, compared with the easier notions of the maxims. 
I think that this improvised and emergent approach characterizes not only stylistics teaching, but also stylistics as a practice: just as it is intermediate between literary studies and linguistics, so its useful terminology is intermediate between the rhetorical terminology of literary studies and the theoretical terminology of linguistics. Stylisticians often pick and choose which elements of a linguistic theory they think will work effectively, and they are happy to combine incompatible theoretical components with each other because they work well as ways of getting at texts. As teachers of linguistics and literature, we must always decide where we stand on any terminological issue. Here is an example. In the stylistics literature (e.g. Leech and Short 1981), a distinction is drawn between various different ways of representing in a fiction a character's thoughts or speech. 'Direct' representations quote the speech or thought; 'indirect' representations re-state the speech or thought from the narrator's (temporal, spatial) perspective. 'Free indirect' representations mix the two of these, giving the effect of a third-person narration that is intermixed with the character's perspective (common in the nineteenth-century novel from Jane Austen onwards). We might treat these as distinct theoretical terms. Although I use them in teaching, I think of them as useful terms, and in addition, I use the theoretical term 'metarepresentation'. From the perspective of a theory of metarepresentations, a sentence that represents another sentence (e.g. what someone says or thinks) is a metarepresentation, and there is no theoretical distinction between a metarepresentation that very closely resembles the original (as in direct speech) and a metarepresentation that less closely resembles the original (as in indirect or free indirect speech). I teach the students the useful terms, because it gives them a rich descriptive vocabulary, and when they run into trouble in a particular text (which blurs the boundaries), this too is interesting. But I also teach the notion of 'metarepresentation' that replaces these terms; while I would not always teach high theory, in this case the full theory is easy to understand, relatively independent of other notions, and very useful in general as a way of thinking about literature. It is a judgement call: we make them all the time when we choose a linguistic terminology for the literature classroom.

However, there is another reason to keep various different types of terminology in play. Rhetorical terms (and perhaps useful terms) are part of what we know about literature, and enter into the inferences we draw about literary texts. When a writer uses a particular type of metaphor, it may be with the intention that that specific type should be recognized and take its place in the processing of implicatures communicated by the text (e.g. as a way of saying 'I am literature'). Furthermore, just as explicit cultural notions may reflect modular thinking (e.g. a verbal taxonomy may reflect the way the taxonomic module works), so rhetorical terms may provide some insight into the working of literature-specific modules. And if, as I suggested earlier, the language of a literary text already has an 
uncertain relation to the language faculty, then the absolute value of theoretical terminology is called into question. Perhaps principles of the language faculty such as 'last resort' (permitting movement of a syntactic constituent only when it is required) are just irrelevant to Pope's literary practice, if Pope is constructing sentences by concatenating phrases together in whatever order serves other formal purposes. It is similarly worth noting that most of the syntactic rules for poetry can be stated in terms of 'school grammar', relating to the surface of the text, and often framed in terms of constructions (i.e. descriptive terms not delivered by current generative approaches).

\section{'Choice' and the Practice of Stylistics}

The same explicature (explicit meaning) can be derived from different sentences. Thus, for example, a specific eventuality can be represented by an active sentence, a passive sentence, or a noun phrase. The participants might be explicitly denoted - by a full noun phrase or by a pronoun or not denoted (as, for example, in a passive sentence where the agent is unexpressed). If it takes place in the past, the sentence might be in past tense or in non-past tense (i.e. 'conversational historic present'); indications of modality or evidentiality can be added, or not added; the sentence might bring out the extent to which the eventuality is an activity or an accomplishment or an achievement; various parts of the sentence might be put into focus (e.g. by order or intonation). The sentence can be modified in these ways, without fundamentally altering its explicit meaning (although some aspects of meaning, and information structure are altered thereby). Much work in stylistics has emphasized the ways in which writers - particularly writers of literary texts - make functionally driven stylistic choices of this kind. In fact, Halliday's 'systemic grammar' (Halliday and Martin 1981), which has been an important theoretical framework for much British and Australian stylistics, places the notion of choice within a system at the heart of the theory.

To illustrate, I cite a jocular British folk tale (the full text is in Briggs 1991). I use it as the basis of an exercise in Fabb (1997). It tells the story of a bunch of idiots who decide to capture a cuckoo and to imprison it in a hedge (without a roof), because they believe that the presence of a cuckoo magically guarantees the harvest. The story ends like this, with my numbers added at boundaries between clauses: "Now", say they, "Us'll have three harvests this year. Look how the hedge be a-growing!" [1] Cuckoo were growing too. [2] Well, the hedge grew nice and high, and the cuckoo grew his wings, [3] and he flied nice and high. And he went!' At [1], the tense changes from non-past (conversational historic present) to past without changing the time reference; at [2], the aspect changes from imperfective (progressive) to perfective while describing essentially the same occurrences; and there is a change in inherent aspect, 
where the sentences represent more or less the same events but with different inherent aspects - the actions up to [2] are activities, after [2] are accomplishments, and after [3] are achievements. The shifts in tense, aspect and inherent aspect do not really correlate with significant differences in what is being represented; instead, these would be largely understood under a stylistics analysis as different choices of how to represent the events of the story. Why does the story shift stylistically in this way at the end? In part, because the story is an extended joke, and the punch line must emerge quickly: the rapid changes perhaps create this effect (more generally, these kinds of weakly motivated change often mark boundaries in a text, of this kind). The shifts themselves, from present imperfective activity to past perfective accomplishment and then achievement, represent symbolically the way in which the cuckoo overcomes the static and repetitive world of the idiots, who live in an eternal foolish present (next year, presumably, they will try the same again). But this is at the level of interpretation; this meaning is not encoded into the sentence by the tenses, or aspects.

\section{What Are the Components of a Literary Linguistics Education?}

In discussing the areas that might be covered in the teaching of linguistics to literature students, I return to the tripartite division with which I began - into (a) the language faculty, (b) central processes involving inference, analogy, etc., and (c) specialized rules for governing literary forms.

The language faculty includes syntax/semantics, the lexicon, and phonology, and perhaps other components, such as a distinct morphology.

- Syntactic structure: I am not convinced that the study of literature requires a highly theorized account of syntactic structure. If students can identify major overt constituents (particularly noun phrases, and the broad structure of the sentence, along even traditional lines), they will be able to make good literary use of this skill, for example, in understanding how enjambment works, or understanding parallelism, or structural ambiguity, or the organization of periodic sentences and the paratactic-hypotactic distinction. Some knowledge of the distinction between grammatical roles (subject, object) and thematic roles (agent, patient) is useful, particularly for those kinds of stylistics that emphasize how alternative formulations of a sentence (i.e. 'transitivity' distinctions) can reveal or conceal components of an eventuality. The tense-aspectmodal systems are relatively easy to teach (at least for English), and are of relevance for understanding various literary processes. In particular, tense is important in narrative; aspect is important for the study of metarepresentational processes, such as the representation of speech and thought; and modality is important for certain genres (such as ghost stories and detective fiction). 
- Phonology: literature students need to know what a syllable is, its structure, and some basic facts about consonants and vowels (including the feature breakdown of these sounds). There is some reason to think that some understanding of levels of phonological representation is relevant (e.g. some sound patterning is based on the output of lexical, some on post-lexical rules, and sometimes underlying forms are also relevant). The link between linguistics and Parisian structuralism can also be clarified by an understanding of some historical issues in phonology (such as distinctive features and archiphonemes).

- The lexicon and morphology: the organization of lexical items into collocations or 'semantic fields' is a fundamental feature of many literary texts. Relations of synonymy and antonymy are important for parallelism. Names have a special status, as do epithets and other periphrastic modes of naming. The 'classic' examples for discussing morphology in the literature classroom involve texts with neologisms such as Carroll's 'Jabberwocky', and there are many other similar examples (I prefer Finnegans Wake) involving either new words formed by affixation or by compounding. Otherwise, an understanding of morphology can help a student recognize parallelisms and patterns in a text (involving morphologically similar words), and relates also to matters of tense and aspect.

Pragmatics is the study of the ways in which central processes, such as inferencing operate. Here, the central goal is to ensure that students understand that communication 'takes place at a risk' because it depends on pragmatic as well as coded (language faculty) processes, and that this is possible because there are "ways in which a statement implies the truth of certain other statements' (Austin 1975: 47), by implicature, entailment and presupposition. In practice, I find that it is easy enough to get students to understand the basic point, but much harder to teach them how to model actual inferential processes. It is possible to teach simple rules such as modus ponens, which is one of the engines of inferencing, but difficult on the one hand to produce anything revealing by working at this level of detail, and also difficult to get my literature students, who otherwise know no logic, to write modus ponens rules correctly. As with various other aspects of 'literary linguistics', we might expect too much of literature students (particularly in writing rules and writing out structural descriptions) when we have not already taught them how to be linguists. Other areas in general pragmatics that are relevant for literary studies include metaphor and other forms of figurative language, and irony and related practices (traditional notions such as 'metaphor' and 'irony' may be better thought of as 'rhetorical' notions rather than theoretical ones), Irony is one of a range of literary devices, including representation of speech and thought (see above), and what literary scholars call 'focalization' (where events are viewed via a character) that are rather simply explained via the notions of metarepresentation and propositional attitude. Metaphor 
has been subject to various quite different ways of explanation in linguistics, some of which look to the pragmatics (e.g. older relevance-theoretic accounts in terms of interpretive resemblance), some of which look to the lexicon (e.g. newer relevance-theoretic accounts of broadening and narrowing of encyclopaedic entries; Wison and Carston 2006).

If there are specialized cognitive modules which are relevant to literature, different modules might perform the following tasks.

- Division of a text into hierarchically organized sections, such as lines, various kinds of superline (the couplet, Langzeile), stanzas, etc. Evidence that this is a specialized module rather than just a general process of dividing any material into sections comes from various cross-linguistic generalizations, and indeed, the striking fact that all literatures in all languages appear to include verse (text divided into lines). Section boundaries often coincide with linguistic constituent boundaries or are otherwise marked by linguistically explainable phenomena (such as rhyme). Lines whose boundaries do not coincide with linguistic constituents (e.g. in enjambment) are particularly interesting, as there is clearly some sensitivity to linguistic structure - whether syntactic or prosodic - so that although divisions 'violate' constituency, they do not do so at random.

- Assignment of a meter to a line of verse. Fabb and Halle (2008) present a theory of how this is achieved, using rules which generate a grid from the line of verse, which is then subject to conditions. Learning about meter involves also learning about syllables (in English), learning about stress, and learning about vowels and consonants - in order to understand why some syllables (e.g. vowel-final before vowel-initial) can be ignored by the metrical rules. Metrical theory is particularly difficult for students, partly because the relevant intuitions seem to be difficult to access: in my experience, they find it difficult to describe rhythmic patterns (e.g. working out which syllable in a polysyllable has the greatest stress). Metrics is an interesting area for literary linguistics, because there are a number of competing linguistic accounts. Although underplayed in much current literature teaching, this is also an area which has been traditionally well-described (we inherit a wealth of 'rhetorical terms' for describing metrical phenomena) and, indeed, has been subject to quite sophisticated pre-linguistic theorizing: think of English poetic analysis around 1600, or Snorri Sturluson's analysis of Icelandic poetic form in his thirteenth-century Edda.

- The identification of parallelism, usually involving syntactically similar structures with different (but related) words. Parallelism shows similar characteristics across a range of literatures, and is very widespread: an indication that it might involve module-specific types of formal description. A basic grounding in syntactic analysis is adequate to identify many cases of parallelism. It is worth looking at how parallelism interacts with 
other types of literary form, such as lineation (parallelism seems in fact to work against some kinds of form, such as meter).

- The division of a narrative into functionally distinct sections, such as orientation (setting the stage), complication and resolution, and coda (bringing the narrative back to the moment of narration). These notions are similar to those found in traditional analysis, but were decisively theorized by Labov and Waletzky (1997) in their analysis of oral narratives. This type of formal description is not particularly linguistic as such: it may hold for all kinds of narrative, whether verbal or non-verbal, and the particular relevance for linguistics is in Labov and Waletzky's demonstration that the boundary between complication and resolution can be marked by stylistic choices (such as repetition, etc.) of the kind discussed earlier, which do not alter the propositional content. A similar point can be made about a rather different principle of organization, where a narrative is divided into episodes (perhaps in a module distinct from the complication-resolution form); as Hymes (1981) has shown, episode boundaries are similarly marked by specific stylistic choices when the stories are told in language.

- The identification of sound patterning, such as rhyme and alliteration. This is an opportunity to teach literature students about consonants and vowels, and syllable structure (because rhyme and alliteration each focus on a specific sub-part of the syllable). Two negative goals are important here: to persuade the students that letters and sounds do not fully correspond (i.e. church and cat and cinema do not alliterate), and that sound symbolism is a pragmatic and contextually driven effect and not a coded association of sound and meaning. I favor non-literary but creative examples such as tongue twisters (great for getting students to understand the feature-based relation between similar sounds), or nonEnglish examples such as the Welsh cynghanedd system (where sequences of consonants are repeated between halves of the line).

- The assignment of texts to genres. In Fabb (2002), I claim that this must be understood as a central, rather than modularized, process. Thus, for example, I suggest that a text is a sonnet not as a fact about the text which somehow inheres in the text itself, but instead by virtue of the text's providing evidence for the thought 'this text is a sonnet', where this thought is derived by inferential procedures (mainly modus ponens) which depend on evidence provided by the text, in the context of general knowledge about sonnets. Thus, if the text has 14 lines (evidence provided by the text) and we know that a sonnet has 14 lines, then this is one reason for thinking that the text is a sonnet. The more reasons, the stronger the evidence for the thought that 'this text is a sonnet'. I think that it is worth speculating on whether there are specialized processes which enable us to identify the genres of texts - again, because generic identification seems similar cross-linguistically, even down to the genres which exist, and also because all literatures seem to require generic 
identification. There are areas of linguistics which have paid particular attention to genre identification; for example, the theory of 'register' in systemic linguistics, or the theory of 'ways of speaking' (Hymes 1989). Language may also provide key evidence for genre identification, and so again there is a link between linguistics and literature.

\section{Conclusion}

It is possible for linguistics and literary studies to share the notion of 'language'. Linguists have specialist knowledge of the language faculty, without which language and hence literature is impossible. But some aspects of literary language are best described not by appeal to the language faculty, but in terms of general and central processes involving inference, resemblance or concatenation, or in terms of specialized (literary-)formal mechanisms. These latter aspects of literary study, involving both form and meaning, are more characteristically in the domain of literary theory, but here too linguists can have a role in clarifying questions and formulating explanations.

\section{Acknowledgements}

Thanks to my Strathclyde University students, to Maya Honda, and for their very useful comments, to Sharon Klein and an anonymous reviewer.

\section{Short Biography}

Nigel Fabb is Professor of Literary Linguistics at the University of Strathclyde. $\mathrm{He}$ is an editor of Journal of Linguistics, and editor, author or co-author of nine books, of which the most recent are (with Mairi Blackings) $A$ Grammar of Ma'di, and (with Morris Halle) Meter in Poetry: a New Theory.

\section{Note}

* Correspondence address: Nigel Fabb, Department of English Studies, University of Strathclyde, 26 Richmond Street, Glasgow G1 1XH, UK. E-mail: n.fabb@strath.ac.uk.

\section{Works Cited}

Ashbery, John. (ed.) 1974. Penguin modern poets: K. Elmslie, K. Koch, J. Schuyler. London, UK: Penguin.

Austin, J. L. 1975. How to do things with words, 2nd edn. Oxford, UK: Oxford University Press. Austin, Timothy R. 1984. Language crafted. A linguistic theory of poetic syntax. Bloomington, IN: Indiana University Press.

Borges, Jorge Luis. 1998. The library of Babel. (first published 1944) Collected fictions, ed. by Jorge Luis Borges and trans. by Andrew Hurley, 112-18. London, UK: Allen Lane.

Boyer, Pascal. 2001. Religion explained. The human instincts that fashion gods. spirits and ancestors. New York, NY: Basic Books. 
Briggs, Katharine M. 1991. A dictionary of British folk-tales in the English language. Part A folk narratives. London, UK: Routledge.

Carruthers, Peter. 2006. The architecture of the mind. Oxford, UK: Oxford University Press.

Fabb, Nigel. 1995. The density of response: a problem for literary criticism and cognitive science. Linguistic Approaches to Literature: papers in literary stylistics. (Discourse Analysis Monographs 17), ed. by J. Payne and J. Wheatley, 143-57. Birmingham, UK: University of Birmingham.

- 1997. Linguistics and literature: language in the verbal arts of the world. Oxford, UK: Blackwell.

Cambridge, UK: Cambridge University Press.

- 2004. Form as fiction. Belgian Journal of English Language and Literatures New series $2.63-73$.

Fabb, Nigel, and Morris Halle. 2008. Meter in poetry: a new theory. Cambridge, UK: Cambridge University Press.

Fodor, Jerry. 1983. The modularity of mind. Cambridge, MA: MIT Press.

- 2000. The mind doesn't work that way. Cambridge, MA: MIT Press.

Halliday, M. A. K., and J. R. Martin. (ed.) 1981. Readings in systemic linguistics. London, UK: Batsford.

Hymes, Dell. 1981. Essays in Native American ethnopoetics. Philadelphia, PA: University of Pennsylvania Press.

- 1989. Ways of speaking. Explorations in the ethnography of speaking, ed. by R. Bauman and J. Sherzer, 433-51. Cambridge, UK: Cambridge University Press.

Labov, William, and Joshua Waletzky. 1997. Narrative Analysis. Oral Versions of Personal Experience. First published in 1967. Oral versions of personal experience: three decades of narrative analysis. Journal of narrative and life history (volume 7, Special Issue), ed. by Michael G.W. Bamberg, 1-14. London, UK: Lawrence Erlbaum.

Leech, G., and M. Short. 1981. Style in fiction. A linguistic introduction to English fictional prose. London, UK: Longman.

Mithen, Steven. 1996. The prehistory of the mind. A search for the origins of art, religion and science. London, UK: Thames and Hudson.

Murphy, Gregory L. 2002. The big book of concepts. Cambridge, MA: MIT Press.

Rothenberg, Jerome, and Pierre Joris. (eds.) 1998. Poems for the millennium. Berkeley, CA: University of California Press.

Ruthven, K. K. 1969. The conceit. London, UK: Methuen.

Sperber, Dan. 1985. On anthropological knowledge. Cambridge, UK: Cambridge University Press.

- 1996. Explaining culture. A naturalistic approach. Oxford, UK: Blackwell.

Sperber, Dan. (ed.) 2000. Metarepresentations. A multidisciplinary perspective. New York, NY: Oxford University Press.

Sperber, Dan, and Deirdre Wilson. 1995. Relevance: communication and cognition, 2nd edn. Oxford, UK: Blackwell.

Thorne, James Peter. 1965. Stylistics and generative grammars. Journal of Linguistics 1.49-59.

Tooby, John, and Leda Cosmides. 1992. The psychological foundations of culture. The adapted mind. Evolutionary psychology and the generation of culture, ed. by Jerome H. Barkow, Leda Cosmides, and John Tooby, 19-136. Oxford, UK: Oxford University Press.

Turner, Mark. 1996. The literary mind. The origins of thought and language. Oxford, UK: Oxford University Press.

Wilson, D., and R. Carston. 2006. Metaphor, relevance and the 'emergent property' issue. Mind and Language 21(3).404-33. 


\section{Please correct and return this set}

Please use the proof correction marks shown below for all alterations and corrections. If you wish to return your proof by fax you should ensure that all amendments are written clearly in dark ink and are made well within the page margins.

\begin{tabular}{|c|c|c|}
\hline Instruction to printer & Textual mark & Marginal mark \\
\hline Leave unchanged & ... under matter to remain & ( ) \\
\hline $\begin{array}{l}\text { Insert in text the matter } \\
\text { indicated in the margin }\end{array}$ & $h$ & $\begin{array}{l}\text { New matter followed by } \\
h \text { or } h \otimes\end{array}$ \\
\hline Delete & $\begin{array}{l}\text { I through single character, rule or underline } \\
\text { or }\end{array}$ & $\sigma$ or $\sigma / \otimes$ \\
\hline $\begin{array}{l}\text { Substitute character or } \\
\text { substitute part of one or } \\
\text { more word(s) }\end{array}$ & / through letter or & $\begin{array}{l}\text { new character / or } \\
\text { new characters / }\end{array}$ \\
\hline Change to italics & — under matter to be changed & $\leftarrow$ \\
\hline Change to capitals & $\equiv$ under matter to be changed & $\equiv$ \\
\hline Change to small capitals & $=$ under matter to be changed & $=$ \\
\hline Change to bold type & $\sim$ under matter to be changed & $\sim$ \\
\hline Change to bold italic & $\approx$ under matter to be changed & $\underline{s i n}$ \\
\hline Change to lower case & Encircle matter to be changed & $\not$ \\
\hline Change italic to upright type & (As above) & \\
\hline Change bold to non-bold type & (As above) & \\
\hline Insert 'superior' character & $\begin{array}{l}\text { I through character or } \\
K \text { where required }\end{array}$ & $\begin{array}{l}y^{\prime} \text { or } y \\
\text { under character } \\
\text { e.g. } y^{2} \text { or } y^{2}\end{array}$ \\
\hline Insert 'inferior' character & (As above) & $\begin{array}{l}\text { L } \\
\text { over character } \\
\text { e.g. } \hat{\imath}\end{array}$ \\
\hline Insert full stop & (As above) & $\odot$ \\
\hline Insert comma & (As above) & , \\
\hline Insert single quotation marks & (As above) & $\begin{array}{l}\dot{y} \text { or } \dot{X} \text { and/or } \\
\dot{y} \text { or } \dot{X}\end{array}$ \\
\hline Insert double quotation marks & (As above) & $\begin{array}{l}\dddot{y} \text { or } \ddot{x} \text { and/or } \\
\ddot{y} \text { or } \ddot{x}\end{array}$ \\
\hline Insert hyphen & (As above) & $1-1$ \\
\hline Start new paragraph & $\digamma$ & 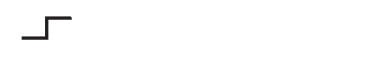 \\
\hline No new paragraph & $\infty$ & $\omega$ \\
\hline Transpose & $\sqcup$ & ᄃ \\
\hline Close up & linking $\bigcirc$ characters & \\
\hline $\begin{array}{l}\text { Insert or substitute space } \\
\text { between characters or words }\end{array}$ & $\begin{array}{l}\text { I through character or } \\
\Lambda \text { where required }\end{array}$ & \\
\hline $\begin{array}{l}\text { Reduce space between } \\
\text { characters or words }\end{array}$ & $\begin{array}{l}\text { between characters or } \\
\text { words affected }\end{array}$ & $\uparrow$ \\
\hline
\end{tabular}

